\title{
Conformable Fractional Partial Differentiation on $n$-Dimensional Time Scales
}

\author{
Nazlı Yazıcı Gözütok* and Uğur Gözütok \\ Department of Mathematical, Karadeniz Technical University \\ 61080 Trabzon, Turkey \\ *Corresponding author: nazliyazici@ktu.edu.tr
}

Article history

Received: 10 May 2018

Received in revised form: 25 March 2019

Accepted: 1 May 2019

Published online: 1 August 2019

\begin{abstract}
In this study, the notion of a conformable partial differentiation of order $\alpha$ on time scales is introduced. Some properties of this new concept are given and relation between the conformable partial differentiation on time scales and the classical partial delta differentiation is revealed.
\end{abstract}

Keywords Calculus on time scales; fractional calculus; conformable partial derivation.

Mathematics Subject Classification 26A33, 26B12, 26E70.

\section{Introduction}

Recently, fractional calculus is one of the most attracted field of mathematics. The purpose of the field is to generalize the ordinary differentiation and integration to non integer order. In accordance with this purpose, several definitions of a differentiation of fractional order is given by various mathematicians, including Riemann-Liouville and Caputo. The theory has advanced many disciplines including signal processing, diffusion problems and wave problems. Studies about fractional calculus could be found in [1-7].

In 2014, a new definition of the fractional derivative called conformable fractional derivative was given by the authors Khalil et al. [8]. Their definition was developed in [9, 10]. In 2016, a natural extension of the conformable fractional derivative to time scales theory was given by the authors Benkhettou et al. [11]. Also, this study extended the time scale calculus to fractional time scale calculus. The authors showed that the fractional calculus on the time scale $\mathbb{T}=[0, \infty)$ corresponds the conformable fractional calculus in [8]. Recently, studies related fractional calculus on time scales is also found in [12-15]. All these developments motivate us to investigate the fractional partial differentiability on time scales.

In this paper, we introduce the notion of a conformable partial differentiation of order $\alpha$ on time scales, give some properties of the concept, and reveal the relation between the conformable partial differentiation on time scales and the classical partial delta differentiation. 


\section{Preliminaries}

We would like to recall some necessary concepts from the calculus on time scales [16]. A time scale $\mathbb{T}$ is an arbitrary nonempty closed subset of the real numbers $\mathbb{R}$. For $t$ in $\mathbb{T}$, the forward jump operator is the function $\sigma: \mathbb{T} \rightarrow \mathbb{T}$ defined by $\sigma(t)=\inf \{s \in \mathbb{T} \mid s>t\}$ and the backward jump operator is the function $\rho: \mathbb{T} \rightarrow \mathbb{T}$ defined by $\rho(t)=\sup \{s \in \mathbb{T} \mid s<t\}$. For $t \in \mathbb{T}$, if $\sigma(t)>t$, then $t$ is called right-scattered; if $\rho(t)<t$, then $t$ is called left-scattered. Further, if $t<\sup \mathbb{T}$ and $\sigma(t)=t$, then $t$ is called right-dense; if $t>\inf \mathbb{T}$ and $\rho(t)=t$, then $t$ is called left-dense. The graininess function $\mu: \mathbb{T} \rightarrow[0, \infty)$ is defined by $\mu(t)=\sigma(t)-t$ for all $t \in \mathbb{T}$. For the function $f: \mathbb{T} \rightarrow \mathbb{R}$, the forward shift $f^{\sigma}: \mathbb{T} \rightarrow \mathbb{R}$ is defined by $f^{\sigma}(t)=f(\sigma(t))$ for all $t \in \mathbb{T}$. The set $\mathbb{T}^{\kappa}$ is defined by

$$
\mathbb{T}^{\kappa}= \begin{cases}\mathbb{T} \backslash(\rho(\sup \mathbb{T}), \sup \mathbb{T}], & \text { sup } \mathbb{T}<\infty \\ \mathbb{T}, & \text { otherwise. }\end{cases}
$$

The differentiability of a function of one variable is given by the following definition.

Definition 1 [16] Assume that $f: \mathbb{T} \rightarrow \mathbb{R}$ is a function and let $t \in \mathbb{T}$. We define $f^{\Delta}(t)$ to be the number, provided it exists, with the property that for any $\epsilon>0$, there exists a neighbourhood $U$ of $t, U=(t-\delta, t+\delta) \cap \mathbb{T}$ for some $\delta>0$, such that

$$
\left|f^{\sigma}(t)-f(s)-f^{\Delta}(t)(\sigma(t)-s)\right| \leq \epsilon|\sigma(t)-s|
$$

for all $s \in U . f^{\Delta}(t)$ is called the delta or Hilger derivative of $f$ at $t$.

It is easily seen that for $\mathbb{T}=\mathbb{R}$, the delta derivative corresponds to the classical derivative. The authors Benkhettou et al. extended this definition to the fractional order.

Definition 2 [1]] Let $f: \mathbb{T} \rightarrow \mathbb{R}, t \in \mathbb{T}^{\kappa}$, and $\alpha \in(0,1]$. For $t>0$, we define $T_{\alpha} f(t)$ to be the number, provided it exists, with the property that given any $\epsilon>0$, there is a $\delta$-neighbourhood $\nu_{t} \subset \mathbb{T}$ of $t, \delta>0$, such that

$$
\left|\left(f^{\sigma}(t)-f(s)\right) t^{1-\alpha}-T_{\alpha} f(t)(\sigma(t)-s)\right| \leq \epsilon|\sigma(t)-s|
$$

for all $s \in \nu_{t} . T_{\alpha} f(t)$ is called the conformable fractional derivative of $f$ of order $\alpha$ at $t$, and the conformable fractional derivative at 0 is defined as $T \alpha f(0)=\lim _{t \rightarrow 0^{+}} T_{\alpha} f(t)$.

One can see that for $\alpha=1$, Definition 2 corresponds to the delta derivative of time scales, i.e. Definition 1. Also, if $\mathbb{T}=\mathbb{R}$, than Definition 2 corresponds to the conformable fractional derivative introduced in [8].

\section{Fractional Differentiation on Time Scales for the Functions of Sev- eral Variables}

For the theory of the functions of several variables on time scales, firstly, we would like to give some necessary definitions and properties. For each $i=1,2, \ldots, n$, let $\mathbb{T}_{i}$ be a time scale, then the set

$$
\mathfrak{T}^{n}=\mathbb{T}_{1} \times \cdots \times \mathbb{T}_{n}=\left\{t=\left(t_{1}, \ldots, t_{n}\right) \mid t_{i} \in \mathbb{T}_{i}, i=1,2, \ldots, n\right\}
$$


is called an $n$-dimensional time scale. If we equip $\mathfrak{T}^{n}$ with the metric

$$
d(t, s)=\left(\sum_{i=1}^{n}\left|t_{i}-s_{i}\right|^{2}\right)^{1 / 2}
$$

for $t, s \in \mathfrak{T}^{n}$, then for the metric space $\left(\mathfrak{T}^{n}, d\right)$, we have all fundamental properties on $\mathfrak{T}^{n}$, including open sets, neighbourhoods, limits, continuity, and so on. For each $i=1,2, \ldots, n$, let $\sigma_{i}$ be the forward jump operator in $\mathbb{T}_{i}$, then the forward jump operator $\sigma: \mathfrak{T}^{n} \rightarrow \mathbb{R}^{n}$ in $\mathfrak{T}^{n}$ is defined by

$$
\sigma(t)=\left(\sigma_{1}\left(t_{1}\right), \ldots, \sigma_{n}\left(t_{n}\right)\right)
$$

for all $t \in \mathfrak{T}^{n}$. In the same way we can define the backward jump operator $\rho: \mathfrak{T}^{n} \rightarrow \mathbb{R}^{n}$ in $\mathfrak{T}^{n}$ as

$$
\rho(t)=\left(\rho_{1}\left(t_{1}\right), \ldots, \rho_{n}\left(t_{n}\right)\right)
$$

for all $t \in \mathfrak{T}^{n}$. For $x=\left(x_{1}, x_{2}, \ldots, x_{n}\right) \in \mathbb{R}^{n}$ and $y=\left(y_{1}, y_{2}, \ldots, y_{n}\right) \in \mathbb{R}^{n}, x \geq y$ iff $x_{i} \geq y_{i}$ for all $i=1,2, \ldots, n$. In the same way, we can write $x>y, x<y$, and $x \leq y$.

Definition 3 [16] Let $t \in \mathfrak{T}^{n}, t=\left(t_{1}, t_{2}, \ldots, t_{n}\right)$.

i. If $\sigma(t)>t$, then $t$ is called strictly right-scattered.

ii. If $\sigma(t) \geq t$ and there are $j, l \in\{1,2, \ldots, n\}$ such that $\sigma_{j}\left(t_{j}\right)>t_{j}$ and $\sigma_{l}\left(t_{l}\right)>t_{l}$, then $t$ is called right-scattered.

iii. If $t<\sup \mathfrak{T}^{n}$ and $\sigma(t)=t$, then $t$ is called right-dense.

iv. If $\rho(t)<t$, then $t$ is called strictly left-scattered.

v. If $\rho(t) \leq t$ and there are $j, l \in\{1,2, \ldots, n\}$ such that $\rho_{j}\left(t_{j}\right)>t_{j}$ and $\rho_{l}\left(t_{l}\right)>t_{l}$, then $t$ is called left-scattered.

vi. If $t>\inf \mathfrak{T}^{n}$ and $\rho(t)=t$, then $t$ is called left-dense.

The graininess function $\mu: \mathfrak{T}^{n} \rightarrow[0, \infty)^{n}$ is defined by

$$
\mu(t)=\left(\mu_{1}\left(t_{1}\right), \ldots, \mu_{n}\left(t_{n}\right)\right)
$$

for all $t \in \mathfrak{T}^{n}$. For a function $f: \mathfrak{T}^{n} \rightarrow \mathbb{R}$, following equalities are given:

$$
\begin{aligned}
f^{\sigma}(t) & =f\left(\sigma_{1}\left(t_{1}\right), \ldots, \sigma_{n}\left(t_{n}\right)\right), \\
f_{i}^{\sigma_{i}}(t) & =f\left(t_{1}, \ldots, t_{i-1}, \sigma_{i}\left(t_{i}\right), t_{i+1}, \ldots, t_{n}\right)
\end{aligned}
$$

for all $t \in \mathfrak{T}^{n}$. Also, the set $\mathfrak{T}^{\kappa n}$ is defined by

$$
\mathfrak{T}^{\kappa n}=\mathbb{T}_{1}^{\kappa} \times \cdots \times \mathbb{T}_{n}^{\kappa}
$$

and the set $\mathfrak{T}^{\kappa_{i} n}$ is defined by

$$
\mathfrak{T}^{\kappa_{i} n}=\mathbb{T}_{1} \times \cdots \times \mathbb{T}_{i-1} \times \mathbb{T}_{i}^{\kappa} \times \mathbb{T}_{i+1} \times \cdots \times \mathbb{T}_{n}
$$

for each $i=1,2, \ldots, n$. Now, we are ready to introduce the notion of the conformable fractional partial differentiability. 
Definition 4 Let $f: \mathfrak{T}^{n} \rightarrow \mathbb{R}, t \in \mathfrak{T}_{i}^{\kappa_{i} n}$, and $\alpha \in(0,1]$. For $t_{i}>0$, we define $\frac{\partial^{\alpha} f}{\Delta_{i} t_{i}^{\alpha}}(t)$ to be the number, provided it exists, with the property that for any $\epsilon>0$, there exists a neighbourhood $U=\left(t_{i}-\delta, t_{i}+\delta\right) \cap \mathbb{T}_{i}$, for some $\delta>0$, such that

$$
\begin{aligned}
\mid\left(f\left(t_{1}, \ldots t_{i-1}, \sigma_{i}\left(t_{i}\right), t_{i+1}, \ldots, t_{n}\right)\right. & \left.-f\left(t_{1}, \ldots, t_{i-1}, s_{i}, t_{i+1}, \ldots, t_{n}\right)\right) t_{i}^{1-\alpha} \\
& -\frac{\partial^{\alpha} f}{\Delta_{i} t_{i}^{\alpha}}(t)\left(\sigma_{i}\left(t_{i}\right)-s_{i}\right)|\leq \epsilon| \sigma_{i}\left(t_{i}\right)-s_{i} \mid
\end{aligned}
$$

for all $s_{i} \in U$. We call $\frac{\partial^{\alpha} f}{\Delta_{i} t_{i}^{\alpha}}(t)$ the conformable partial derivative of $f$ of order $\alpha$ with respect to $t_{i}$ at $t$. We say that $f$ is conformable partial differentiable of order $\alpha$ with respect to $t_{i}$ in $\mathfrak{T}_{i}^{\kappa_{i} n}$ if $\frac{\partial^{\alpha} f}{\Delta_{i} t_{i}^{\alpha}}(t)$ exists for all $t \in \mathfrak{T}_{i}^{\kappa_{i} n}$.

Remark 1 If $\alpha=1$, then we obtain by Definition 4 the partial delta derivative or partial Hilger derivative of $f$ with respect to $t_{i}$ at $t$.

Remark 2 For $t \in \mathbb{T}^{n}$ and $s_{i} \in \mathbb{T}_{i}$, if we write

$$
\begin{aligned}
t_{s_{i}} & =\left(t_{1}, \ldots, t_{i-1}, s_{i}, t_{i+1}, \ldots, t_{n}\right), \\
f_{i}^{\sigma_{i}}(t) & =f\left(t_{1}, \ldots, t_{i-1}, \sigma_{i}\left(t_{i}\right), t_{i+1}, \ldots, t_{n}\right), i=1,2, \ldots, n,
\end{aligned}
$$

then we can rewrite (3) as

$$
\left|\left(f_{i}^{\sigma_{i}}(t)-f\left(t_{s_{i}}\right)\right) t_{i}^{1-\alpha}-\frac{\partial^{\alpha} f}{\Delta_{i} t_{i}^{\alpha}}(t)\left(\sigma_{i}\left(t_{i}\right)-s_{i}\right)\right| \leq \epsilon\left|\sigma_{i}\left(t_{i}\right)-s_{i}\right| .
$$

Example 1 Let the function $f: \mathfrak{T}^{3} \rightarrow \mathbb{R}$ be defined by $f(t)=t_{1}^{2}+4 t_{2} t_{3}$. For $t_{1}>0$, we will show that $\frac{\partial^{1 / 2} f}{\Delta_{1} t_{1}^{1 / 2}}(t)=t_{1}^{1 / 2} \sigma_{1}\left(t_{1}\right)+t_{1}^{3 / 2}$.

For any $\epsilon>0$, there exists a number $\delta, 0<\delta \leq \frac{\epsilon}{t_{1}^{1 / 2}}$ such that for any $s_{1} \in\left(t_{1}-\delta, t_{1}+\delta\right) \cap \mathbb{T}_{1}$. Since $t_{1}-\delta<s_{1}<t_{1}+\delta$, it is obtained that $-\delta<t_{1}-s_{1}<\delta$ giving $\left|t_{1}-s_{1}\right|<\delta \leq \frac{\epsilon}{t_{1}^{1 / 2}}$. Hence,

$$
\begin{aligned}
& \left|\left(f_{i}^{\sigma_{i}}(t)-f\left(t_{s_{i}}\right)\right) t_{i}^{1 / 2}-\frac{\partial^{1 / 2} f}{\Delta_{i} t_{i}^{1 / 2}}(t)\left(\sigma_{i}\left(t_{i}\right)-s_{i}\right)\right| \\
& =\left|\left(f\left(\sigma_{1}\left(t_{1}\right), t_{2}, t_{3}\right)-f\left(s_{1}, t_{2}, t_{3}\right)\right) t_{1}^{1 / 2}-\left(t_{1}^{1 / 2} \sigma_{1}\left(t_{1}\right)+t_{1}^{3 / 2}\right)\left(\sigma_{1}\left(t_{1}\right)-s_{1}\right)\right| \\
& =\left|\left(\sigma_{1}^{2}\left(t_{1}\right)+4 t_{2} t_{3}-s_{1}^{2}-4 t_{2} t_{3}\right) t_{1}^{1 / 2}-t_{1}^{1 / 2}\left(\sigma_{1}\left(t_{1}\right)+t_{1}\right)\left(\sigma_{1}\left(t_{1}\right)-s_{1}\right)\right| \\
& =t_{1}^{1 / 2}\left|\left(\sigma_{1}\left(t_{1}\right)-s_{1}\right)\left(\sigma_{1}\left(t_{1}\right)+s_{1}\right)-\left(\sigma_{1}\left(t_{1}\right)+t_{1}\right)\left(\sigma_{1}\left(t_{1}\right)-s_{1}\right)\right| \\
& =t_{1}^{1 / 2}\left|\left(\sigma_{1}\left(t_{1}\right)-s_{1}\right)\left[\left(\sigma_{1}\left(t_{1}\right)+s_{1}\right)-\left(\sigma_{1}\left(t_{1}\right)+t_{1}\right)\right]\right| \\
& =t_{1}^{1 / 2}\left|t_{1}-s_{1}\right|\left|\sigma_{1}\left(t_{1}\right)-s_{1}\right|<\delta t_{1}^{1 / 2}\left|\sigma_{1}\left(t_{1}\right)-s_{1}\right| \leq \epsilon\left|\sigma_{1}\left(t_{1}\right)-s_{1}\right| .
\end{aligned}
$$

Theorem 1 Let $f: \mathfrak{T}^{n} \rightarrow \mathbb{R}$ be a function, $t \in \mathfrak{T}_{i}^{\kappa_{i} n}, \alpha \in(0,1]$, and $t_{i}>0$. If $f$ is conformable partial differentiable of order $\alpha$ with respect to $t_{i}$ at $t$, then

$$
\lim _{s_{i} \rightarrow t_{i}} f\left(t_{s_{i}}\right)=f(t) .
$$


Proof Since $f$ is conformable partial differentiable of order $\alpha$ with respect to $t_{i}$ at $t$, we have that for any $\epsilon>0$, there exist a number $\delta>0, \delta<\min \{1, \gamma\}$, such that for any $s_{i} \in\left(t_{i}-\delta, t_{i}+\delta\right) \cap \mathbb{T}_{i}$ we have

$$
\left|\left(f_{i}^{\sigma_{i}}(t)-f\left(t_{s_{i}}\right)\right) t_{i}^{1-\alpha}-\frac{\partial^{\alpha} f}{\Delta_{i} t_{i}^{\alpha}}(t)\left(\sigma_{i}\left(t_{i}\right)-s_{i}\right)\right| \leq \gamma\left|\sigma_{i}\left(t_{i}\right)-s_{i}\right|,
$$

and

for $\gamma=\frac{\epsilon t_{i}^{1-\alpha}}{1+2 \mu_{i}\left(t_{i}\right)+\left|\frac{\partial^{\alpha} f}{\Delta_{i} t_{i}^{\alpha}}(t)\right|}$.

$$
\left|\left(f_{i}^{\sigma_{i}}(t)-f(t)\right) t_{i}^{1-\alpha}-\frac{\partial^{\alpha} f}{\Delta_{i} t_{i}^{\alpha}}(t)\left(\sigma_{i}\left(t_{i}\right)-t_{i}\right)\right| \leq \gamma\left|\sigma_{i}\left(t_{i}\right)-t_{i}\right|
$$

Hence, for every $s_{i} \in\left(t_{i}-\delta, t_{i}+\delta\right) \cap \mathbb{T}_{i}$ we get

$$
\begin{aligned}
\left|f(t)-f\left(t_{s_{i}}\right)\right| & =\mid\left(f_{i}^{\sigma_{i}}(t)-f\left(t_{s_{i}}\right)\right)-\frac{\partial^{\alpha} f}{\Delta_{i} t_{i}^{\alpha}}(t)\left(\sigma_{i}\left(t_{i}\right)-s_{i}\right) t_{i}^{\alpha-1} \\
& -\left[\left(f_{i}^{\sigma_{i}}(t)-f(t)\right)-\frac{\partial^{\alpha} f}{\Delta_{i} t_{i}^{\alpha}}(t)\left(\sigma_{i}\left(t_{i}\right)-t_{i}\right) t_{i}^{\alpha-1}\right]+\frac{\partial^{\alpha} f}{\Delta_{i} t_{i}^{\alpha}}(t)\left(t_{i}-s_{i}\right) t_{i}^{\alpha-1} \mid \\
& \leq\left|\left(f_{i}^{\sigma_{i}}(t)-f\left(t_{s_{i}}\right)\right)-\frac{\partial^{\alpha} f}{\Delta_{i} t_{i}^{\alpha}}(t)\left(\sigma_{i}\left(t_{i}\right)-s_{i}\right) t_{i}^{\alpha-1}\right| \\
& +\left|\left(f_{i}^{\sigma_{i}}(t)-f(t)\right)-\frac{\partial^{\alpha} f}{\Delta_{i} t_{i}^{\alpha}}(t)\left(\sigma_{i}\left(t_{i}\right)-t_{i}\right) t_{i}^{\alpha-1}\right| \\
& +\left|\frac{\partial^{\alpha} f}{\Delta_{i} t_{i}^{\alpha}}(t)\right|\left|\left(t_{i}-s_{i}\right)\right| t_{i}^{\alpha-1} \\
& \leq \gamma\left|\sigma_{i}\left(t_{i}\right)-s_{i}\right| t_{i}^{\alpha-1}+\gamma\left|\sigma_{i}\left(t_{i}\right)-t_{i}\right| t_{i}^{\alpha-1}+\left|\frac{\partial^{\alpha} f}{\Delta_{i} t_{i}^{\alpha}}(t)\right|\left|\left(t_{i}-s_{i}\right)\right| t_{i}^{\alpha-1} \\
& \leq \gamma\left|\sigma_{i}\left(t_{i}\right)-s_{i}\right| t_{i}^{\alpha-1}+\gamma \mu_{i}\left(t_{i}\right) t_{i}^{\alpha-1}+\gamma\left|\frac{\partial^{\alpha} f}{\Delta_{i} t_{i}^{\alpha}}(t)\right| t_{i}^{\alpha-1} \\
& =\gamma\left(\mu_{i}\left(t_{i}\right)+\left|\sigma_{i}\left(t_{i}\right)-s_{i}\right|+\left|\frac{\partial^{\alpha} f}{\Delta_{i} t_{i}^{\alpha}}(t)\right|\right) t_{i}^{\alpha-1} \\
& =\gamma\left(\mu_{i}\left(t_{i}\right)+\left|\sigma_{i}\left(t_{i}\right)-t_{i}+t_{i}-s_{i}\right|+\left|\frac{\partial^{\alpha} f}{\Delta_{i} t_{i}^{\alpha}}(t)\right|\right) t_{i}^{\alpha-1} \\
& \leq \gamma\left(2 \mu_{i}\left(t_{i}\right)+\left|t_{i}-s_{i}\right|+\left|\frac{\partial^{\alpha} f}{\Delta_{i} t_{i}^{\alpha}}(t)\right|\right) t_{i}^{\alpha-1} \\
& \leq \gamma\left(1+2 \mu_{i}\left(t_{i}\right)+\left|\frac{\partial^{\alpha} f}{\Delta_{i} t_{i}^{\alpha}}(t)\right|\right) t_{i}^{\alpha-1}=\epsilon .
\end{aligned}
$$

Theorem 2 Let $f: \mathfrak{T}^{n} \rightarrow \mathbb{R}, t \in \mathfrak{T}_{i}^{\kappa_{i} n}, \alpha \in(0,1], t_{i}>0$, and

$$
\lim _{s_{i} \rightarrow t_{i}} f\left(t_{s_{i}}\right)=f(t) .
$$

If $t_{i}<\sigma_{i}\left(t_{i}\right)$, then $f$ is conformable partial differentiable of order $\alpha$ with respect to $t_{i}$ at $t$ and

$$
\frac{\partial^{\alpha} f}{\Delta_{i} t_{i}^{\alpha}}(t)=\frac{f_{i}^{\sigma_{i}}(t)-f(t)}{\mu_{i}\left(t_{i}\right)} t_{i}^{1-\alpha}
$$


Proof As $s_{i} \rightarrow t_{i}$, by using (3) and $\lim _{s_{i} \rightarrow t_{i}} f\left(t_{s_{i}}\right)=f(t)$, we get

$$
\frac{\partial^{\alpha} f}{\Delta_{i} t_{i}^{\alpha}}(t)=\frac{f_{i}^{\sigma_{i}}(t)-f(t)}{\mu_{i}\left(t_{i}\right)} t_{i}^{1-\alpha}
$$

Example 2 Let $h>0, \mathbb{T}_{1}=h \mathbb{Z}^{+}$and $\mathbb{T}_{2}=3^{\mathbb{N}}$. Let $\mathfrak{T}^{2}=h \mathbb{Z}^{+} \times 3^{\mathbb{N}}$ and define $f: \mathfrak{T}^{2} \rightarrow \mathbb{R}$ by

$$
f(t)=3 t_{1}^{2}+t_{1} t_{2}, \quad t=\left(t_{1}, t_{2}\right) \in \mathfrak{T}^{2} .
$$

We have $\sigma_{1}\left(t_{1}\right)=t_{1}+h, t_{1} \in h \mathbb{Z}$ and $\sigma_{2}\left(t_{2}\right)=3 t_{2}, t_{2} \in 3^{\mathbb{N}}$. Therefore, $t_{1}<\sigma_{1}\left(t_{1}\right)$ and $t_{2}<\sigma_{2}\left(t_{2}\right)$. By Theorem 2, we have

$$
\frac{\partial^{1 / 2} f}{\Delta_{1} t_{1}^{1 / 2}}(t)=\frac{f_{1}^{\sigma_{1}}(t)-f(t)}{\mu_{1}\left(t_{1}\right)} t_{1}^{1 / 2}=6 t_{1}^{3 / 2}+t_{1}^{1 / 2} t_{2}+3 h t_{1}^{1 / 2},
$$

and

$$
\frac{\partial^{1 / 2} f}{\Delta_{2} t_{2}^{1 / 2}}(t)=\frac{f_{2}^{\sigma_{2}}(t)-f(t)}{\mu_{2}\left(t_{2}\right)} t_{2}^{1 / 2}=t_{1} t_{2}^{1 / 2} .
$$

Theorem 3 Let $t \in \mathfrak{T}_{i}^{\kappa_{i} n}, t_{i}=\sigma_{i}\left(t_{i}\right)>0$, and $\alpha \in(0,1]$. Then $f$ is conformable partial differentiable of order $\alpha$ with respect to $t_{i}$ at $t$ if and only if the limit $\lim _{s_{i} \rightarrow t_{i}} \frac{f(t)-f\left(t_{s_{i}}\right)}{t_{i}-s_{i}} t_{i}^{1-\alpha}$ exists as a finite number. In this case,

$$
\frac{\partial^{\alpha} f}{\Delta_{i} t_{i}^{\alpha}}(t)=\lim _{s_{i} \rightarrow t_{i}} \frac{f(t)-f\left(t_{s_{i}}\right)}{t_{i}-s_{i}} t_{i}^{1-\alpha}
$$

Proof Let $f$ be conformable partial differentiable of order $\alpha$ with respect to $t_{i}$ at $t$. Then for any $\epsilon>0$, there exists $\delta>0$ such that for any $s_{i} \in\left(t_{i}-\delta, t_{i}+\delta\right) \cap \mathbb{T}_{i}$, we have (4). Since $\sigma_{i}\left(t_{i}\right)=t_{i}$, we get

$$
\left|\frac{f(t)-f\left(t_{s_{i}}\right)}{t_{i}-s_{i}} t_{i}^{1-\alpha}-\frac{\partial^{\alpha} f}{\Delta_{i} t_{i}^{\alpha}}(t)\right| \leq \epsilon
$$

Then we have

$$
\frac{\partial^{\alpha} f}{\Delta_{i} t_{i}^{\alpha}}(t)=\lim _{s_{i} \rightarrow t_{i}} \frac{f(t)-f\left(t_{s_{i}}\right)}{t_{i}-s_{i}} t_{i}^{1-\alpha}
$$

On the other hand, let

$$
\frac{\partial^{\alpha} f}{\Delta_{i} t_{i}^{\alpha}}(t)=\lim _{s_{i} \rightarrow t_{i}} \frac{f(t)-f\left(t_{s_{i}}\right)}{t_{i}-s_{i}} t_{i}^{1-\alpha}
$$

Then for any $\epsilon>0$, there exists a number $\delta>0$ such that for any $s_{i} \in\left(t_{i}-\delta, t_{i}+\delta\right) \cap \mathbb{T}_{i}$ we have

$$
\left|\left(f(t)-f\left(t_{s_{i}}\right)\right) t_{i}^{1-\alpha}-\frac{\partial^{\alpha} f}{\Delta_{i} t_{i}^{\alpha}}(t)\left(t_{i}-s_{i}\right)\right| \leq \epsilon\left|t_{i}-s_{i}\right|
$$

Since $\sigma_{i}\left(t_{i}\right)=t_{i}$

$$
\left|\left(f_{i}^{\sigma_{i}}(t)-f\left(t_{s_{i}}\right)\right) t_{i}^{1-\alpha}-\frac{\partial^{\alpha} f}{\Delta_{i} t_{i}^{\alpha}}(t)\left(\sigma_{i}\left(t_{i}\right)-s_{i}\right)\right| \leq \epsilon\left|\sigma_{i}\left(t_{i}\right)-s_{i}\right| .
$$

Remark 3 If we consider the case $\mathfrak{T}^{n}=\mathbb{R}^{n}$, then Theorem 3 corresponds to the conformable fractional derivative introduced in [9]. 


\section{Some Properties of the Conformable Partial Derivative}

Theorem 4 Let $t \in \mathfrak{T}_{i}^{\kappa_{i} n}, t_{i}>0$, and $\alpha \in(0,1]$. Let $f: \mathfrak{T}^{n} \rightarrow \mathbb{R}$ be a function that is conformable partial differentiable of order $\alpha$ with respect to $t_{i}$. If $c \in \mathbb{R}$, then $c f$ is conformable partial differentiable of order $\alpha$ with respect to $t_{i}$, and

$$
\frac{\partial^{\alpha}(c f)}{\Delta_{i} t_{i}^{\alpha}}(t)=c \frac{\partial^{\alpha} f}{\Delta_{i} t_{i}^{\alpha}}(t)
$$

Proof Assume that $c \neq 0$. Since $f$ is conformable partial differentiable of order $\alpha$ with respect to $t_{i}$, for any $\epsilon>0$, there exists $\delta>0$ such that for any $s_{i} \in\left(t_{i}-\delta, t_{i}+\delta\right) \cap \mathbb{T}_{i}$, we have

$$
\left|\left(f_{i}^{\sigma_{i}}(t)-f\left(t_{s_{i}}\right)\right) t_{i}^{1-\alpha}-\frac{\partial^{\alpha} f}{\Delta_{i} t_{i}^{\alpha}}(t)\left(\sigma_{i}\left(t_{i}\right)-s_{i}\right)\right| \leq \frac{\epsilon}{|c|}\left|\sigma_{i}\left(t_{i}\right)-s_{i}\right| .
$$

Hence, we have

$$
\begin{aligned}
& \left|\left((c f)_{i}^{\sigma_{i}}(t)-(c f)\left(t_{s_{i}}\right)\right) t_{i}^{1-\alpha}-c \frac{\partial^{\alpha} f}{\Delta_{i} t_{i}^{\alpha}}(t)\left(\sigma_{i}\left(t_{i}\right)-s_{i}\right)\right| \\
& =|c|\left|\left(f_{i}^{\sigma_{i}}(t)-f\left(t_{s_{i}}\right)\right) t_{i}^{1-\alpha}-\frac{\partial^{\alpha} f}{\Delta_{i} t_{i}^{\alpha}}(t)\left(\sigma_{i}\left(t_{i}\right)-s_{i}\right)\right| \leq \epsilon\left|\sigma_{i}\left(t_{i}\right)-s_{i}\right| .
\end{aligned}
$$

Example 3 Let $f(t)=t_{1} t_{2} t_{3}, t=\left(t_{1}, t_{2}, t_{3}\right) \in \Lambda^{3}$ and $\frac{\partial^{1 / 2} f}{\Delta_{1} t_{1}^{1 / 2}}(t)=t_{1}^{1 / 2} t_{2} t_{3}$. We will show that $\frac{\partial^{1 / 2}(3 f)}{\Delta_{1} t_{1}^{1 / 2}}(t)=3 t_{1}^{1 / 2} t_{2} t_{3}$. For any $\epsilon>0$, there exists $\delta>0$ such that for every $s_{1} \epsilon$ $\left(t_{1}-\delta, t_{1}+\delta\right) \cap \mathbb{T}_{1}$, we have

$$
\begin{aligned}
& \left|\left((3 f)\left(\sigma_{1}\left(t_{1}\right), t_{2}, t_{3}\right)-(3 f)\left(s_{1}, t_{2}, t_{3}\right)\right) t_{1}^{1 / 2}-3 t_{1}^{1 / 2} t_{2} t_{3}\left(\sigma_{1}\left(t_{1}\right)-s_{1}\right)\right| \\
& =\left|\left(3 \sigma_{1}\left(t_{1}\right) t_{2} t_{3}-3 s_{1} t_{2} t_{3}\right) t_{1}^{1 / 2}-3 t_{1}^{1 / 2} t_{2} t_{3}\left(\sigma_{1}\left(t_{1}\right)-s_{1}\right)\right| \\
& =3 t_{1}^{1 / 2}\left|t_{2} t_{3}\left(\sigma_{1}\left(t_{1}\right)-s_{1}-\sigma_{1}\left(t_{1}\right)+s_{1}\right)\right|=0 \leq \epsilon\left|\sigma_{1}\left(t_{1}\right)-s_{1}\right| .
\end{aligned}
$$

Theorem 5 Let $t \in \mathfrak{T}_{i}^{\kappa_{i} n}, t_{i}>0$, and $\alpha \in(0,1]$. If $f, g: \mathfrak{T}^{n} \rightarrow \mathbb{R}$ are conformable partial differentiable of order $\alpha$ with respect to $t_{i}$, then $f+g$ is conformable partial differentiable of order $\alpha$ with respect to $t_{i}$, and

$$
\frac{\partial^{\alpha}(f+g)}{\Delta_{i} t_{i}^{\alpha}}(t)=\frac{\partial^{\alpha} f}{\Delta_{i} t_{i}^{\alpha}}(t)+\frac{\partial^{\alpha} g}{\Delta_{i} t_{i}^{\alpha}}(t)
$$

Proof The proof is straightforward.

Example 4 Let $\Lambda^{2}=\mathbb{N} \times \mathbb{N}$ and define $h: \Lambda^{2} \rightarrow \mathbb{R}$ by $h(t)=t_{1}^{2}+2 t_{1} t_{2},\left(t_{1}, t_{2}\right) \in \Lambda^{2}$. We will find $\frac{\partial^{1 / 2} h}{\Delta_{1} t_{1}^{1 / 2}}(t)$ for $t \in \Lambda_{1}^{\kappa_{1} 2}$. Let $f(t)=t_{1}^{2}, g(t)=2 t_{1} t_{2}, t \in \Lambda^{2}$. Here $\mathbb{T}_{1}=\mathbb{T}_{2}=\mathbb{N}$ and 
$\sigma_{1}\left(t_{1}\right)=t_{1}+1, t_{1} \in \mathbb{T}_{1}$. We have

$$
\begin{aligned}
\frac{\partial^{1 / 2} f}{\Delta_{1} t_{1}^{1 / 2}}(t) & =t_{1}^{1 / 2} \sigma_{1}\left(t_{1}\right)+t_{1}^{3 / 2} \\
& =2 t_{1}^{3 / 2}+t_{1}^{1 / 2} \\
\frac{\partial^{1 / 2} g}{\Delta_{1} t_{1}^{1 / 2}}(t)=2 t_{1}^{1 / 2} t_{2} . &
\end{aligned}
$$

Thus we have

$$
\begin{aligned}
\frac{\partial^{1 / 2} h}{\Delta_{1} t_{1}^{1 / 2}}(t) & =\frac{\partial^{1 / 2} f}{\Delta_{1} t_{1}^{1 / 2}}(t)+\frac{\partial^{1 / 2} g}{\Delta_{1} t_{1}^{1 / 2}}(t) \\
& =2 t_{1}^{3 / 2}+t_{1}^{1 / 2}+2 t_{1}^{1 / 2} t_{2} .
\end{aligned}
$$

Theorem 6 Let $t \in \mathfrak{T}_{i}^{\kappa_{i} n}, t_{i}>0$, and $\alpha \in(0,1]$. If $f, g: \mathfrak{T}^{n} \rightarrow \mathbb{R}$ are conformable partial differentiable of order $\alpha$ with respect to $t_{i}$, then $\mathrm{fg}$ is conformable partial differentiable of order $\alpha$ with respect to $t_{i}$, and

$$
\frac{\partial^{\alpha}(f g)}{\Delta_{i} t_{i}^{\alpha}}(t)=\frac{\partial^{\alpha} f}{\Delta_{i} t_{i}^{\alpha}}(t) g(t)+f_{i}^{\sigma_{i}}(t) \frac{\partial^{\alpha} g}{\Delta_{i} t_{i}^{\alpha}}(t)
$$

Proof Since $f$ and $g$ are conformable partial differentiable of order $\alpha$ with respect to $t_{i}$, for any $\epsilon>0$ there exists a number $\delta>0$ such that for any $s_{i} \in\left(t_{i}-\delta, t_{i}+\delta\right) \cap \mathbb{T}_{i}$ we have

$$
\begin{aligned}
& \left|\left(f_{i}^{\sigma_{i}}(t)-f\left(t_{s_{i}}\right)\right) t_{i}^{1-\alpha}-\frac{\partial^{\alpha} f}{\Delta_{i} t_{i}^{\alpha}}(t)\left(\sigma_{i}\left(t_{i}\right)-s_{i}\right)\right| \leq \gamma\left|\sigma_{i}\left(t_{i}\right)-s_{i}\right|, \\
& \left|\left(g_{i}^{\sigma_{i}}(t)-g\left(t_{s_{i}}\right)\right) t_{i}^{1-\alpha}-\frac{\partial^{\alpha} g}{\Delta_{i} t_{i}^{\alpha}}(t)\left(\sigma_{i}\left(t_{i}\right)-s_{i}\right)\right| \leq \gamma\left|\sigma_{i}\left(t_{i}\right)-s_{i}\right|,
\end{aligned}
$$

where $\gamma<$

$$
\begin{aligned}
& 1+\left|\frac{\partial^{\alpha} f}{\Delta_{i} t_{i}^{\alpha}}(t)\right|+\left|g\left(t_{s_{i}}\right)\right|+\left|f_{i}^{\sigma_{i}}(t)\right| \\
& \left|\left((f g)_{i}^{\sigma_{i}}(t)-(f g)\left(t_{s_{i}}\right)\right) t_{i}^{1-\alpha}-\left(\frac{\partial^{\alpha} f}{\Delta_{i} t_{i}^{\alpha}}(t) g(t)+f_{i}^{\sigma_{i}}(t) \frac{\partial^{\alpha} g}{\Delta_{i} t_{i}^{\alpha}}(t)\right)\left(\sigma_{i}\left(t_{i}\right)-s_{i}\right)\right| \\
& =\mid f_{i}^{\sigma_{i}}(t)\left[\left(g_{i}^{\sigma_{i}}(t)-g\left(t_{s_{i}}\right)\right) t_{i}^{1-\alpha}-\frac{\partial^{\alpha} g}{\Delta_{i} t_{i}^{\alpha}}(t)\left(\sigma_{i}\left(t_{i}\right)-s_{i}\right)\right] \\
& +\left(f_{i}^{\sigma_{i}}(t) g\left(t_{s_{i}}\right)-f\left(t_{s_{i}}\right) g\left(t_{s_{i}}\right)\right) t_{i}^{1-\alpha}-\frac{\partial^{\alpha} f}{\Delta_{i} t_{i}^{\alpha}}(t) g(t)\left(\sigma_{i}\left(t_{i}\right)-s_{i}\right) \mid \\
& =\mid f_{i}^{\sigma_{i}}(t)\left[\left(g_{i}^{\sigma_{i}}(t)-g\left(t_{s_{i}}\right)\right) t_{i}^{1-\alpha}-\frac{\partial^{\alpha} g}{\Delta_{i} t_{i}^{\alpha}}(t)\left(\sigma_{i}\left(t_{i}\right)-s_{i}\right)\right] \\
& +g\left(t_{s_{i}}\right)\left[\left(f_{i}^{\sigma_{i}}(t)-f\left(t_{s_{i}}\right)\right) t_{i}^{1-\alpha}-\frac{\partial^{\alpha} f}{\Delta_{i} t_{i}^{\alpha}}(t)\left(\sigma_{i}\left(t_{i}\right)-s_{i}\right)\right] \\
& +\left(g\left(t_{s_{i}}\right)-g(t)\right) \frac{\partial^{\alpha} f}{\Delta_{i} t_{i}^{\alpha}}(t)\left(\sigma_{i}\left(t_{i}\right)-s_{i}\right) \mid \\
& \leq \gamma\left(1+\left|\frac{\partial^{\alpha} f}{\Delta_{i} t_{i}^{\alpha}}(t)\right|+\left|g\left(t_{s_{i}}\right)\right|+\left|f_{i}^{\sigma_{i}}(t)\right|\right)\left|\sigma_{i}\left(t_{i}\right)-s_{i}\right|<\epsilon\left|\sigma_{i}\left(t_{i}\right)-s_{i}\right| .
\end{aligned}
$$


Example 5 Let $\Lambda^{2}=\mathbb{N} \times \mathbb{N}_{0}^{2}$ and define $h: \Lambda^{2} \rightarrow \mathbb{R}$ by $h(t)=\left(t_{1}^{2}+2 t_{1}\right)\left(t_{1}^{3}+t_{2}\right), t \in \Lambda^{2}$. Here, $\mathbb{T}_{1}=\mathbb{N}$ and $\mathbb{T}_{2}=\mathbb{N}_{0}^{2}$. Then $\sigma_{1}\left(t_{1}\right)=t_{1}+1, t_{1} \in \mathbb{T}_{1}, \sigma_{2}\left(t_{2}\right)=\left(1+t_{2}^{1 / 2}\right)^{2}, t_{2} \in \mathbb{T}_{2}$. We will find $\frac{\partial^{1 / 2} h}{\Delta_{1} t_{1}^{1 / 2}}(t), t \in \Lambda_{1}^{\kappa_{1} 2}$. Let $f(t)=t_{1}^{2}+2 t_{1}, g(t)=t_{1}^{3}+t_{2}, t \in \Lambda^{2}$. Hence, $h(t)=f(t) g(t)$. For $t \in \Lambda_{1}^{\kappa_{1} 2}$ it is obtained

$$
\begin{aligned}
& \frac{\partial^{1 / 2} h}{\Delta_{1} t_{1}^{1 / 2}}(t)=\frac{\partial^{1 / 2} f}{\Delta_{1} t_{1}^{1 / 2}}(t) g(t)+f_{1}^{\sigma_{1}}(t) \frac{\partial^{1 / 2} g}{\Delta_{1} t_{1}^{1 / 2}}(t) \\
& =\left(\sigma_{1}\left(t_{1}\right) t_{1}^{1 / 2}+t_{1}^{3 / 2}+2 t_{1}^{1 / 2}\right) g(t)+\left(\sigma_{1}^{2}\left(t_{1}\right)+2 \sigma_{1}\left(t_{1}\right)\right)\left(\sigma_{1}^{2}\left(t_{1}\right) t_{1}^{1 / 2}+t_{1}^{3 / 2} \sigma_{1}\left(t_{1}\right)+t_{1}^{5 / 2}\right) \\
& =\left(2 t_{1}^{3 / 2}+3 t_{1}^{1 / 2}\right)\left(t_{1}^{3}+t_{2}\right)+\left(t_{1}^{2}+4 t_{1}+3\right)\left(3 t_{1}^{5 / 2}+3 t_{1}^{3 / 2}+t_{1}^{1 / 2}\right) \\
& =5 t_{1}^{9 / 2}+18 t_{1}^{7 / 2}+22 t_{1}^{5 / 2}+13 t_{1}^{3 / 2}+2 t_{1}^{3 / 2} t_{2}+3 t_{1}^{1 / 2} t_{2}+3 t_{1}^{1 / 2}
\end{aligned}
$$

Theorem 7 Let $t \in \mathfrak{T}_{i}^{\kappa_{i} n}, t_{i}>0$, and $\alpha \in(0,1]$. If $f, g: \mathfrak{T}^{n} \rightarrow \mathbb{R}$ are conformable partial differentiable of order $\alpha$ with respect to $t_{i}$ and $g_{i}^{\sigma_{i}}(t) g(t) \neq 0$, then $\frac{f}{g}$ is conformable partial differentiable of order $\alpha$ with respect to $t_{i}$, and

$$
\frac{\partial^{\alpha}\left(\frac{f}{g}\right)}{\Delta_{i} t_{i}^{\alpha}}(t)=\frac{\frac{\partial^{\alpha} f}{\Delta_{i} t_{i}^{\alpha}}(t) g(t)-f(t) \frac{\partial^{\alpha} g}{\Delta_{i} t_{i}^{\alpha}}(t)}{g_{i}^{\sigma_{i}}(t) g(t)} .
$$

Proof Since $f$ and $g$ are conformable partial differentiable of order $\alpha$ with respect to $t_{i}$, for any $\epsilon>0$ there exists a number $\delta>0$ such that for any $s_{i} \in\left(t_{i}-\delta, t_{i}+\delta\right) \cap \mathbb{T}_{i}$ we have

$$
\begin{aligned}
& \left|\left(f_{i}^{\sigma_{i}}(t)-f\left(t_{s_{i}}\right)\right) t_{i}^{1-\alpha}-\frac{\partial^{\alpha} f}{\Delta_{i} t_{i}^{\alpha}}(t)\left(\sigma_{i}\left(t_{i}\right)-s_{i}\right)\right| \leq \gamma\left|\sigma_{i}\left(t_{i}\right)-s_{i}\right|, \\
& \left|\left(g_{i}^{\sigma_{i}}(t)-g\left(t_{s_{i}}\right)\right) t_{i}^{1-\alpha}-\frac{\partial^{\alpha} g}{\Delta_{i} t_{i}^{\alpha}}(t)\left(\sigma_{i}\left(t_{i}\right)-s_{i}\right)\right| \leq \gamma\left|\sigma_{i}\left(t_{i}\right)-s_{i}\right|,
\end{aligned}
$$

where $\gamma<\epsilon \frac{\left|g_{i}^{\sigma_{i}}(t)\right|\left|g\left(t_{s_{i}}\right)\right||g(t)|}{1+|g(t)|\left|g\left(t_{s_{i}}\right)\right|+|g(t)|\left|f\left(t_{s_{i}}\right)\right|+\left|\frac{\partial^{\alpha} g}{\Delta_{i} t_{i}^{\alpha}}(t)\right|}$. Then,

$$
\begin{aligned}
& \left|\left(\left(\frac{f}{g}\right)_{i}^{\sigma_{i}}(t)-\left(\frac{f}{g}\right)\left(t_{s_{i}}\right)\right) t_{i}^{1-\alpha}-\left(\frac{\frac{\partial^{\alpha} f}{\Delta_{i} t_{i}^{\alpha}}(t) g(t)-f(t) \frac{\partial^{\alpha} g}{\Delta_{i} t_{i}^{\alpha}}(t)}{g_{i}^{\sigma_{i}}(t) g(t)}\right)\left(\sigma_{i}\left(t_{i}\right)-s_{i}\right)\right| \\
& =\frac{1}{\left|g_{i}^{\sigma_{i}}(t)\right|\left|g\left(t_{s_{i}}\right)\right||g(t)|} \mid g(t) g\left(t_{s_{i}}\right)\left[\left(f_{i}^{\sigma_{i}}(t)-f\left(t_{s_{i}}\right)\right) t_{i}^{1-\alpha}-\frac{\partial^{\alpha} f}{\Delta_{i} t_{i}^{\alpha}}(t)\left(\sigma_{i}\left(t_{i}\right)-s_{i}\right)\right] \\
& -f\left(t_{s_{i}}\right) g(t)\left[\left(g_{i}^{\sigma_{i}}(t)-g\left(t_{s_{i}}\right)\right) t_{i}^{1-\alpha}-\frac{\partial^{\alpha} g}{\Delta_{i} t_{i}^{\alpha}}(t)\left(\sigma_{i}\left(t_{i}\right)-s_{i}\right)\right] \\
& +\frac{\partial^{\alpha} g}{\Delta_{i} t_{i}^{\alpha}}(t)\left(f(t) g\left(t_{s_{i}}\right)-f\left(t_{s_{i}}\right)-g(t)\right)\left(\sigma\left(t_{i}\right)-s_{i}\right) \mid \\
& \leq \frac{1}{\left|g_{i}^{\sigma_{i}}(t)\right|\left|g\left(t_{s_{i}}\right)\right||g(t)|} \gamma\left(1+|g(t)|\left|g\left(t_{s_{i}}\right)\right|+|g(t)|\left|f\left(t_{s_{i}}\right)\right|+\left|\frac{\partial^{\alpha} g}{\Delta_{i} t_{i}^{\alpha}}(t)\right|\right)\left|\sigma_{i}\left(t_{i}\right)-s_{i}\right| \\
& <\epsilon\left|\sigma_{i}\left(t_{i}\right)-s_{i}\right| .
\end{aligned}
$$


Example 6 Let $\Lambda^{2}=\mathbb{N} \times \mathbb{N}$ and define $h: \Lambda^{2} \rightarrow \mathbb{R}$ by $h(t)=\frac{t_{1}^{2}+2 t_{1} t_{2}+t_{2}^{3}}{t_{1}+t_{2}},\left(t_{1}, t_{2}\right) \in \Lambda^{2}$. We will find $\frac{\partial^{1 / 2} h}{\Delta_{1} t_{1}^{1 / 2}}(t)$ for $t \in \Lambda_{1}^{\kappa_{1} 2}$. Let $f(t)=t_{1}^{2}+2 t_{1} t_{2}+t_{2}^{3}, g(t)=t_{1}+t_{2}, t \in \Lambda^{2}$. Here $\mathbb{T}_{1}=\mathbb{T}_{2}=\mathbb{N}$ and $\sigma_{1}\left(t_{1}\right)=t_{1}+1, t_{1} \in \mathbb{T}_{1}$. We have

$$
\begin{aligned}
\frac{\partial^{1 / 2} f}{\Delta_{1} t_{1}^{1 / 2}}(t) & =t_{1}^{1 / 2} \sigma_{1}\left(t_{1}\right)+t_{1}^{3 / 2}+2 t_{1}^{1 / 2} t_{2} \\
& =2 t_{1}^{3 / 2}+t_{1}^{1 / 2}+2 t_{1}^{1 / 2} t_{2} \\
\frac{\partial^{1 / 2} g}{\Delta_{1} t_{1}^{1 / 2}}(t) & =t_{1}^{1 / 2} \\
g_{1}^{\sigma_{1}} & =t_{1}+t_{2}+1 .
\end{aligned}
$$

Thus we have

$$
\begin{aligned}
\frac{\partial^{1 / 2} h}{\Delta_{1} t_{1}^{1 / 2}}(t) & =\frac{\frac{\partial^{1 / 2} f}{\Delta_{1} t_{1}^{1 / 2}}(t) g(t)-f(t) \frac{\partial^{1 / 2} g}{\Delta_{1} t_{1}^{1 / 2}}(t)}{g_{1}^{\sigma_{1}}(t) g(t)} \\
& =\frac{\left(2 t_{1}^{3 / 2}+t_{1}^{1 / 2}+2 t_{1}^{1 / 2} t_{2}\right)\left(t_{1}+t_{2}\right)-\left(t_{1}^{2}+2 t_{1} t_{2}+t_{2}^{3}\right) t_{1}^{1 / 2}}{\left(t_{1}+t_{2}+1\right)\left(t_{1}+t_{2}\right)} \\
& =\frac{t_{1}^{5 / 2}+t_{1}^{3 / 2}+2 t_{1}^{3 / 2} t_{2}+t_{1}^{1 / 2} t_{2}+2 t_{1}^{1 / 2} t_{2}^{2}-t_{1}^{1 / 2} t_{2}^{3}}{\left(t_{1}+t_{2}+1\right)\left(t_{1}+t_{2}\right)}
\end{aligned}
$$

\section{Conclusion}

In our paper, fractional calculus on multidimensional time scales is investigated by using the most recent notion of the fractional derivative called conformable fractional derivative. Our findings are in agreement with the partial delta derivative on multidimensional time scales and the conformable fractional derivative on $\mathbb{R}^{n}$, and generalize them together. In the future, we will investigate the successive conformable partial differentiation and the completely conformable fractional differentiable functions on multidimensional time scales.

\section{Acknowledgements}

The first author would like to thank TUBITAK(The Scientific and Technological Research Council of Turkey) for their financial supports during her doctorate studies.

\section{References}

[1] Machado,J. T., Kiryakova, V. and Mainardi, F. Recent history of fractional calculus. Communications in Nonlinear Science and Numerical Simulation. 2011. 16(3): 1140-1153. 
[2] Tomar, V. and Maden, E. Set, $(k, s)$-Riemann Liouville fractional integral inequalities for continuous random variables. Arabian Journal of Mathematics. 2017. 6(1): 55-63 .

[3] Nwaeze, E. R. and Torres, D. F. M. Chain rules and inequalities for the BHT fractional calculus on arbitrary timescales. Arabian Journal of Mathematics. 2017. 6(1): 13-20.

[4] Liu, Y. Solvability of impulsive periodic boundary value problems for higher order fractional differential equations. Arabian Journal of Mathematics. 2016. 5(4): 195-214.

[5] Miller, K. and Ross, B. An Introduction to the Fractional Calculus and Fractional Differential Equations. New York: Wiley. 1993.

[6] Tejado, I., Valerio, D. and Valerio, N. Fractional Calculus in Economic Growth Modelling: The Spanish Case, Controlo 2014. In Proceedings of the 11th Portuguese Conference on Automatic Control 321. 449-458. 2015.

[7] Meilanov, R. P. and Magomedov, R. A. Thermodynamics in fractional calculus. Journal of Engineering Physics and Thermophysics. 2014. 87(6): 1521-1531.

[8] Khalil, R., Al Horani, M., Yousef, A. and Sababheh, M. A new definition of fractional derivative. Journal of Computational and Applied Mathematics. 2014. 264: 65-70.

[9] Yazıcı Gözütok, N. and Gözütok, U. Multi-variable conformable fractional calculus, Filomat. 2018. 32(1): 45-53.

[10] Abdeljawad, T. On conformable fractional calculus. Journal of Computational and Applied Mathematics. 2015. 279: 57-66.

[11] Benkhettou, N., Hassani, S. and Torres, D. F. M. A conformable fractional calculus on arbitrary time scales. Journal of King Saud University - Science. 2016. 28(1): 93-98.

[12] Bayour, B., Hammoudi, A. and Torres, D. F. M. A truly conformable calculus on time scales. Global and Stochastic Analysis. arXiv:1705. 08928.

[13] Zhao, D. and You, X. A new fractional derivative on time scales. Advances in Applied Mathematical Analysis. 2016. 11(1): 1-9.

[14] Gülşen, T., Yılmaz, E. and Göktaş, S. Conformable fractional Dirac system on time scales. Journal of Inequalities and Applications. 2017. 161: 1-10.

[15] Gülşen, T. and Yllmaz, E. Spectral theory of Dirac system on time scales. Applicable Analysis. 2016. 96(16): 1-11.

[16] Bohner, M. and Georgiev, S. G. Multivariable Dynamic Calculus on Time Scales. Switzerland: Springer. 2016.

[17] Hilger, S. Analysis on measure chain-a unified approach to continuous and discrete calculus. Results in Mathematics. 1990. 18: 19-56. 